\title{
Cáncer de páncreas. Epidemiología de su mal pronóstico
}

\author{
Dres. Patricia Alejandra López Penza * , Luis Ruso Martínez ${ }^{\dagger}$
}

\section{Resumen}

Introducción: el cáncer de páncreas se caracteriza por su agresividad biológica, mal pronóstico oncológico y diagnóstico tardío. La mayoría con baja tasa de resecabilidad (10\% a $20 \%)$, por la presencia de factores de irresecabilidad al momento del diagnóstico.

Objetivo: descripción epidemiológica de las características del cáncer de páncreas irresecable, los tratamientos paliativos realizados, la sobrevida global y en relación con los procedimientos realizados.

Material y método: estudio descriptivo, multivariable, retrospectivo de los cánceres de páncreas irresecables, de enero de 2010 a diciembre de 2015, en la Clínica Quirúrgica 3 del Hospital Maciel.

Resultados: de los 30 pacientes irresecables: igual distribución por sexo; edad promedio: 61 años, cefálicos en $67 \%$ de los casos. El $53 \%$ adelgazados al momento de la primera consulta; $52 \%$ presentaban compromiso ganglionar locorregional, y $50 \%$ presentaba metástasis hepáticas o ascitis. A 52\% de los pacientes se les realizó algún tipo de derivación biliar; quirúrgica en 53\% (63\% coledocoduodenostomías) y el resto percutánea o endoscópica. El 31\% de los pacientes, por lo avanzado de la enfermedad, no recibió tratamiento quirúrgico ni endoscópico. La sobrevida media del tratamiento paliativo quirúrgico 0 endoscópico fue de 3,7 meses.

Conclusiones: al momento del diagnóstico, la mitad de los casos tenían metástasis y repercusión nutricional severa, con indicadores locorregionales de irresecabilidad o incurabilidad. Un tercio de los casos no recibió tratamiento oncológico por lo avanzado de la enfermedad. La sobrevida global fue escasa. Esto permite sugerir que son imprescindibles medidas sanitarias para su diagnóstico precoz e incremento de la tasa de resecabilidad.

Palabras clave: NEOPLASIAS PANCREÁTICAS

DESVIACIÓN BILIOPANCREÁTICA

STENTS

CUIDADOS PALIATIVOS

PRONÓSTICO

Key words: PANCREATIC NEOPLASMS

BILIOPANCREATIC DIVERSION

STENTS

PALLIATIVE CARE

PROGNOSIS

\footnotetext{
* Asistente de Clínica Quirúrgica 3. Hospital Maciel.

† Profesor de Cirugía. Clínica Quirúrgica 3. Hospital Maciel.

Clínica Quirúrgica 3. Hospital Maciel. Montevideo, Uruguay.

No conflicto de intereses.

Correspondencia: Patricia López Penza. Correo electrónico: patricialopezpenza@hotmail.com

Recibido: 24/4/17

Aprobado: 31/7/17
} 


\section{Introducción}

La incidencia global del cáncer de páncreas es de 10/100.000 habitantes en nuestro país y ocupa el sexto y noveno lugar de mortalidad en la mujer y en el hombre, respectivamente ${ }^{(1)}$. Es decir que se producen entre $350 \mathrm{a}$ 400 nuevos casos/año de este tipo de tumores. El 80\% de estos son adenocarcinomas acinares ${ }^{(2)}$, la mayoría de localización cefálica ${ }^{(3)}$.

Con baja resecabilidad ${ }^{(4-6)}(10 \%$ a $20 \%)$, vinculada a una estricta selección preoperatoria en base a estudios imagenológicos así como a la laparoscopía estadificado$\mathrm{ra}^{(7)}$. En nuestro medio al momento de su diagnóstico la mayoría de los pacientes son candidatos a la paliación de sus síntomas por presentar elementos de irresecabilidad $\mathrm{o}$ incurabilidad.

La paliación de la obstrucción biliar/duodenal, del dolor u otros síntomas es primordial en estos pacientes. Las técnicas paliativas han mejorado la calidad de vida de los pacientes, sin prolongar la sobrevida a mediano ni largo plazo.

El objetivo de esta comunicación es realizar una descripción epidemiológica de las características clínicas y paraclínicas del cáncer de páncreas al momento del diagnóstico, los diferentes tipos de tratamientos paliativos instituidos y sus resultados en términos de sobrevida global.

\section{Material y método}

Estudio descriptivo, retrospectivo, multivariable, que utilizó la base de registro de pacientes neoplásicos de la Clínica Quirúrgica 3 del Hospital Maciel en un período de seis años (enero de 2010 a diciembre de 2015).

Se analizaron las características epidemiológicas clínicas e imagenológicas, el tipo de tratamiento paliativo y la sobrevida global en relación con el tratamiento realizado.

Se incluyeron los pacientes con ictericia obstructiva o anictéricos con diagnóstico topográfico de cáncer de páncreas.

El diagnóstico de cáncer de páncreas se realizó en base a criterios clínicos e imagenológicos a través de la tomografía abdominal con contraste intravenoso. Solo se indicó la biopsia en caso de indicación de quimioterapia.

Se define imagenológicamente por la presencia de un típico nódulo sólido hipodenso respecto al tejido pancreático normal, independientemente de la topografía. Asociado a signos indirectos, como ser la interrupción ductal abrupta, efecto masa, atrofia y dilatación ductal en páncreas distal si su topografía es cefálica ${ }^{(8)}$.

El tratamiento paliativo se consideró en los pacientes que presentaban extenso compromiso locorregional: compromiso de la arteria mesentérica superior, vena porta en más de $180^{\circ}$ de su circunferencia; presencia de enfermedad sistémica: metástasis pulmonares, metástasis hepáticas, carcinomatosis peritoneal (ascitis o nódulos peritoneales) por tomografía en el preoperatorio o hallazgos intraoperatorios.

La presencia de metástasis hepática, carcinomatosis peritoneal, metástasis pulmonares, clasifican al paciente según los estadios de la unidad internacional contra el cáncer (UICC) en estadio IV.

Los tratamientos realizados fueron quirúrgicos, endoscópicos, percutáneos o médicos a cargo del equipo de medicina paliativa.

Las opciones quirúrgicas implicaron derivaciones biliodigestivas (hepaticoyeyunoanastomosis o coledocoduodenostomía) o digestivas (gastroenteroanastomosis) realizadas en forma aislada o concomitantemente. Se utilizó el stent biliar y la derivación biliar externa como procedimiento mínimamente invasivo en pacientes en que las condiciones generales no permitían realizar un procedimiento quirúrgico invasivo.

El seguimiento se realizó mediante los registros clínicos, información del médico tratante y datos solicitados a la Comisión Honoraria de lucha Contra el Cáncer.

En el análisis estadístico de las variables se expresan con valores absolutos y su porcentaje, la edad en promedio y su rango.

La sobrevida se expresa con la curva de Kaplan-Meyer.

\section{Resultados}

De los 30 pacientes, 15 eran mujeres (50\%) y 15 hombres $(50 \%)$. La edad media fue de 61 años (rango: 44 a 79 años).

La tabla 1 muestra las características del cáncer de páncreas al momento del diagnóstico según el sexo.

El 66\% (n: 19) eran cefálicos; 17\% (n: 5) del uncus pancreático; 14\% (n: 4) corporocaudales, y 3\% (n: 1) comprometía todo el órgano.

La ictericia se presentó en $100 \%$ (n: 19) de los tumores cefálicos; en $50 \%$ (n: 2) del uncus pancreático, y en ningún caso de los corporocaudales. Más de la mitad de los casos (53\%) presentaban adelgazamiento en la primera consulta.

La extensión lesional extraglandular se observó como adenopatías regionales $40 \%$ (n: 12 ) de los casos; estas se asociaron en $45 \%$ (n: 5) con metástasis hepática; en $18 \%$ (n: 2) con ascitis, y en $63 \%$ (n: 7) con compromiso venoso.

En el 10\% (n: 3) de los pacientes existió concomitancia de ascitis y metástasis hepática. El compromiso venoso se observó en 37\% (n: 11), el ganglionar fue en un solo caso certificado por anatomía patológica mediante 
Tabla 1. Características del cáncer de páncreas según el sexo.

\begin{tabular}{lccc}
\hline & Hombre & Mujer & Total \\
\hline Topografía & 7 & 12 & 19 \\
Cefálico & 2 & 3 & 5 \\
Uncus & 1 & 2 & 4 \\
Corporocaudal & 1 & 0 & 1 \\
Difuso & & & \\
\hline Tamaño & 4 & 0 & 4 \\
$<3 \mathrm{~cm}$ & 10 & 15 & 25 \\
$\geq 3 \mathrm{~cm}$ & & & \\
\hline Compromiso venoso & 5 & 6 & 11 \\
Sí & & & \\
No & & & \\
\hline
\end{tabular}

Compromiso arterial

\begin{tabular}{llll} 
Sí & 2 & 3 & 5 \\
No & & & \\
\hline
\end{tabular}

Compromiso ganglionar

\begin{tabular}{rrrr} 
Sí & 3 & 9 & 12 \\
No & & & \\
\hline Ascitis & & & \\
Sí & 1 & 4 & 5 \\
No & & & \\
\hline
\end{tabular}

Metástasis hepática

\begin{tabular}{cccc} 
Sí & 4 & 8 & 12 \\
No & & & \\
\hline
\end{tabular}

Nódulos peritoneales

\begin{tabular}{lll} 
Sí & 0 & 0 \\
No & & \\
\hline
\end{tabular}

biopsia extemporánea en el intraoperatorio, lo cual marcó la decisión de un tratamiento paliativo (tabla 2).

El 43\% (n: 13) de la serie corresponde a un estadio IV, según la UICC, secundario a la presencia de metástasis hepática o ascitis.

El $92 \%$ de los pacientes con enfermedad diseminada tenían metástasis hepática y $17 \%$ ascitis.

Un paciente $(10 \%)$ presentaba concomitantemente metástasis hepática, ascitis y metástasis pulmonar.
Tabla 2. Compromiso locorregional y general.

\begin{tabular}{lcc}
\hline \multicolumn{1}{c}{ Características } & \multicolumn{1}{c}{ Sí } & No \\
\hline Compromiso ganglionar & $12(40 \%)$ & $18(60 \%)$ \\
Invasión venosa & $11(37 \%)$ & $19(63 \%)$ \\
Invasión arterial & $5(17 \%)$ & $25(83 \%)$ \\
Solalargia & $9(30 \%)$ & $21(70 \%)$ \\
Metástasis hepática & $12(40 \%)$ & $18(60 \%)$ \\
Ascitis & $5(17 \%)$ & $25(83 \%)$ \\
\hline
\end{tabular}

En cuanto al tratamiento, en $33 \%$ ( n: 10) no se realizó ningún procedimiento invasivo, solo tratamiento médico paliativo a cargo de la Unidad de Cuidados Paliativos del Hospital Maciel, por lo avanzado de la enfermedad.

En $37 \%$ (n: 11) se realizaron procedimientos quirúrgicos: derivaciones biliodigestiva y digestiva. En este grupo se confeccionó en 46\% (n: 5) una coledocoduodenostomía latero lateral y en $27 \%$ (n: 3 ) una hepaticoyeyunostomía, correspondiendo a los tumores de topografía cefálica y del uncus. En 36\% (n: 4) se realizó un procedimiento de derivación digestiva -gastroeneteroanastomosis latero lateral-, en el caso de un tumor del uncus con compresión de DIII y otro a nivel del cuerpo pancreático. A un solo paciente se le realizó una doble derivación.

En 23\% (n: 7) se utilizó algún procedimiento mínimamente invasivo, el de drenaje biliar externo se efectuó en $86 \%$ (n: 6) y la colocación de stent-de drenaje biliar interno- en 14\%. En un paciente se inició el tratamiento con poliquimioterapia.

En relación con el tratamiento del dolor, solo en un caso recibió neurolisis de los nervios esplácnicos por vía laparotómica.

La sobrevida media en los pacientes con tratamiento paliativo fue de 3, meses (111días, rango: 5-540 días) (figura 1).

La sobrevida media de los pacientes metastásicos fue de dos meses (rango: 5-270 días); los que presentaban metástasis hepáticas fue de 1,7 meses, y los localmente avanzado de 4 meses (rango: 46-540 días) (figura $2)$; sin evidencia significativa entre ellos ( $\mathrm{p}:<0,005)$.

\section{Discusión}

En el cáncer de páncreas la oportunidad de control oncológico y de curación está ligada a su diagnóstico en etapa de localización glandular exclusiva y a tumores pequeños. Aun así, no se han desarrollado métodos de screening para su diagnóstico precoz, lo cual genera un 


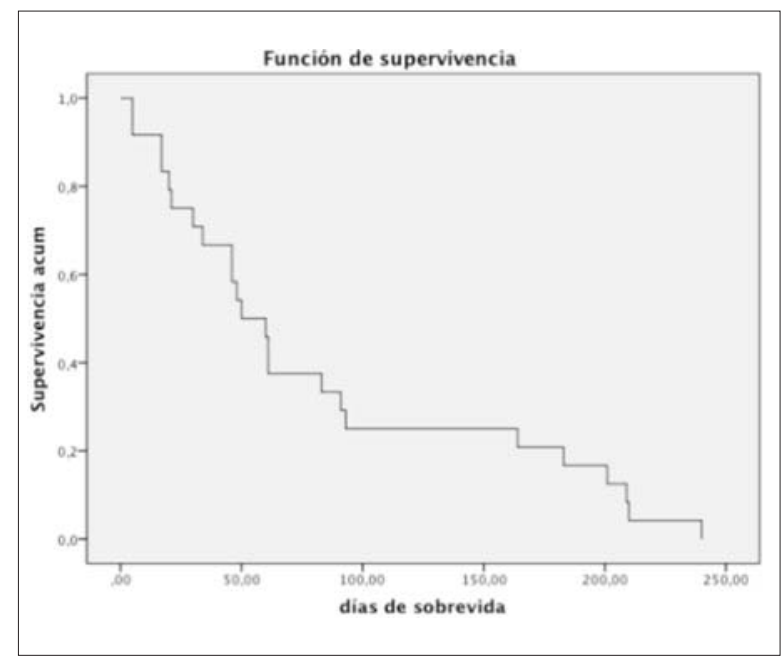

Figura 1. Sobrevida global según Kaplan-Meyer.

impacto negativo con implicancias en la sobrevida a corto y largo plazo.

El tratamiento de los pacientes con cáncer localmente avanzado o metastásico implica un elevado costo sanitario y requiere un enfoque multidisciplinario entre cirujano, gastroenterólogo y oncólogo con el único objetivo posible de paliar los síntomas de obstrucción biliar o duodenal y aliviar el dolor debido a la infiltración del plexo solar.

La historia natural del cáncer de páncreas lo subdivide en localmente avanzado y en enfermedad metastásica.

El presente análisis refleja una realidad devastadora: el $43 \%$ de los casos se diagnostican en estadio IV, es decir con enfermedad metastásica; $92 \%$ a nivel hepático, y $8 \%$ a nivel peritoneal. Es interesante destacar que la localización más frecuente de la enfermedad metastásica fue a nivel hepático (92\%), lo cual difiere de la literatura internacional en que es alrededor de $50 \%{ }^{(9)}$.

La aparición tardía de los síntomas hace que solo entre $10 \%$ y $20 \%{ }^{(4-6)}$ de los pacientes se presenten con una enfermedad pasible de una resección quirúrgica al momento del diagnóstico.

La tasa de resecabilidad de nuestra serie fue de 19\%, valor acorde con la experiencia internacional ${ }^{(10,11)}$.

Y también es concordante que el restante 85\%-90\% se presenta localmente avanzado, no resecables $(40 \%)$ o con enfermedad metastásica (45\%). La resecabilidad no solo se vincula con factores técnico-quirúrgicos o limitantes por la condición general del paciente, sino que además no se recomienda la exéresis paliativa de los adenocarcinomas localmente avanzados de páncreas debido a la alta morbilidad y mortalidad relacionada con el escaso beneficio de sobrevida ${ }^{(12)}$. También los proce-

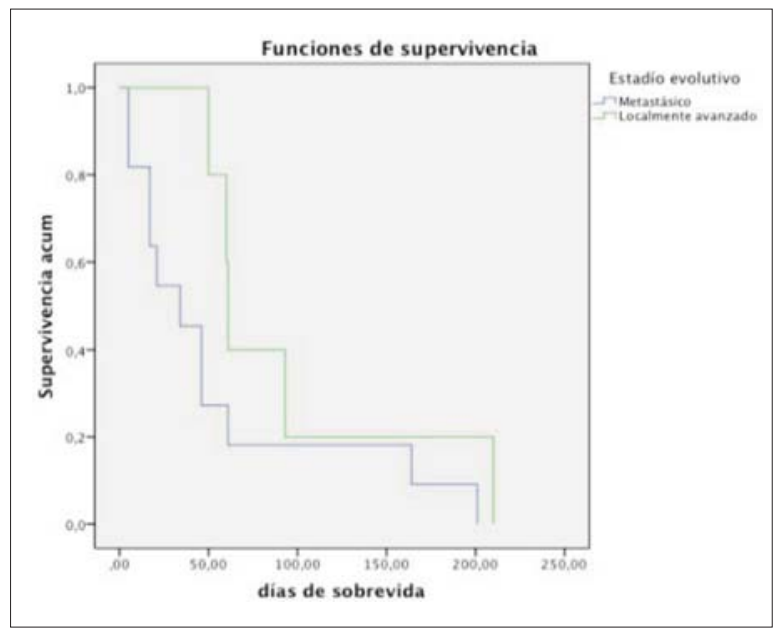

Figura 2. Sobrevida de pacientes metastásicos y los localmente avanzados.

dimientos de bypass paliativos se indican con menos frecuencia. Muller ${ }^{(13)}$, en su serie, informó la utilización global de bypass paliativos en $22 \%$ de los casos.

Este cambio puede atribuirse al uso de la tomografía multislide con reconstrucción vascular, que optimiza la evaluación de la extensión locorregional del tumor y disminuye la tasa de enfermedad extraglandular imprevista en el momento de la exploración.

La carcinomatosis peritoneal-sobre todo a micronódulos- es difícil de establecer por imágenes (TC y RNM), pero es cada vez más importante determinar el beneficio de una derivación paliativa en esta cohorte de pacientes. Por esta razón se ha incrementado el uso sistemático de la laparoscopía estadificadora para evitar laparotomías innecesarias ${ }^{(14)}$.

Existen estudios que demuestran la ventaja de la derivación biliodigestiva como tratamiento paliativo quirúrgico versus stent endoscópico, con menor índice de reobstrucción a favor de la cirugía ${ }^{(15-17)}$.

Sin embargo, ensayos controlados aleatorios ${ }^{(18-20)}$ no encontraron diferencia en el éxito técnico de la tasa en el alivio de la ictericia, la mortalidad y la mediana supervivencia. Además, los pacientes con metástasis a distancia son peores candidatos para la paliación quirúrgica que los pacientes con cáncer localmente avanzado.

El uso de stents plásticos es de gran utilidad para la paliación durante cortos períodos de tiempo, reservándose los metálicos para pacientes sin metástasis ${ }^{(21)}$.

A largo plazo se manifiesta un número significativo de complicaciones, que son mayores con stents plásticos. Al comparase los stents metálicos con los plásticos en períodos prolongados las tasas de reintervención fueron de $29,8 \%$ versus $55,6 \%$ y la hospitalización de $17,9 \%$ vs $27,8 \%$, así como la duración de la permeabili- 
dad, 121 vs 77,3 días ${ }^{(21)}$. Al momento de la elección del tipo de stent debe considerarse que Soderlund ${ }^{(22)}$ demostró que la sobrevida de los pacientes con metástasis a distancia es similar al tiempo de permeabilidad de los stents plásticos. Además, otro problema subyacente es el costo de los stents. El metaanálisis de Moss ${ }^{(23)}$ demostró que el costo de los stents metálicos es 15 a 40 veces más que los plásticos y solo rentable si el paciente sobrevive más de cuatro meses.

Nuestros enfermos recibieron inicialmente una derivación biliar externa por punción transparieto hepática para posteriormente colocación de stent. En la mayoría de los casos $(86 \%$, n: 6) no se pudo colocar en la evolución el stent por la defunción de los pacientes.

La realización de un bypass gastroyeyunal en forma rutinaria es muy controvertida. Si no se realiza la gastroyeyunostomía en el momento de la derivación biliar, $13 \%$ a $21 \%$ requerirán la misma ${ }^{(24,25)}$. Un ensayo controlado aleatorizado confirma que $19 \%$ de los pacientes requieren intervención por obstrucción duodenal cuando son aptos para el procedimiento quirúrgico ${ }^{(26)}$.

Nosotros realizamos derivación gástrica solo en aquellos pacientes con síntomas de estenosis gastroduodenal clínicamente manifiesta o hallazgos endoscópicos de obstrucción duodenal.

Los stents gástricos metálicos autoexpansibles parecen ser una opción más favorable que el bypass quirúrgico para el alivio de la obstrucción gástrica sintomática, con una excelente tasa de éxito, supervivencia similar, estancia hospitalaria más corta y costo mucho más bajo que la derivación quirúrgica ${ }^{(27,28)}$, teniendo en cuenta que en la evaluación del beneficio de cualquier procedimiento quirúrgico la esperanza de vida del paciente es fundamental en la toma de decisiones.

Los pacientes con cáncer localmente avanzado presentaron una sobrevida media de cuatro meses, algo menor que a nivel internacional, que es de 6 a 11 me$\operatorname{ses}^{(29-32)}$.

La sobrevida media de los pacientes metastásicos fue de dos meses, cifra similar a los datos internacionales, que es de tres a seis meses ${ }^{(33-39)}$.

En este sentido debe recordarse que las metástasis hepáticas o peritoneales constituyen un factor pronóstico adverso independiente ${ }^{(19,37)}$; incluso hay estudios que no muestran diferencias en el pronóstico de acuerdo con la localización metastásica ${ }^{(20)}$, mientras que otros asocian la sobrevida en forma significativa e independiente con el sitio inicial de metástasis, con una sobrevida media de 7,5 meses para la enfermedad extrahepática; 4,8 meses en metástasis hepáticas solas, y de 2,4 meses cuando presenta metástasis en ambas topografías ${ }^{(18)}$. En nuestra casuística la sobrevida media de los pacientes con metástasis hepática fue de 1,7 meses, valores significativamente menores que los mencionados por otros autores.

La existencia de solo tres pacientes con enfermedad extrahepática no nos permite un estudio comparativo de la misma.

La escasa sobrevida de estos pacientes, como consecuencia de un diagnóstico en etapas avanzadas de la enfermedad, sea por el compromiso locorregional o metastásico a distancia, nos plantean la necesidad de búsqueda de métodos de screening de esta patología, con el objetivo de diagnóstico en etapas precoces de la enfermedad.

\section{Conclusiones}

Este estudio muestra que el diagnóstico de cáncer de páncreas se realiza en etapas tardías de la enfermedad. En más de la mitad de los casos los pacientes presentan desnutrición en la primera consulta. La mitad de los pacientes tenían metástasis con indicadores locorregionales de irresecabilidad o incurabilidad y un tercio de los casos no recibió tratamiento oncológico invasivo por lo avanzado de la enfermedad. La sobrevida media fue escasa (menor de cuatro meses). Esta situación permite sugerir que es imprescindible implementar medidas sanitarias para el diagnóstico precoz del cáncer de páncreas, lo que podría modificar la evolución natural de la enfermedad en nuestro país.

\section{Abstract}

Introduction: pancreatic cancer is a particularly aggressive cancer with a poor prognosis and it is typically diagnosed at a late stage. Most cases have a lower rate of resectability $(10 \%$ to $20 \%)$ as a result of certain unresectable factors at the time of diagnosis.

Objective: the study aims to provide an epidemiological description of the characteristics of unresectable pancreatic cancer, palliative treatment, global survival and procedures applied.

Method: descriptive, multivariable, retrospective study of cases of unresectable cancer from January 2010 to December 2015, at the $3^{\text {rd }}$ Surgical Unit of Hospital Maciel, Montevideo, Uruguay.

Results: considering the 30 unresectable patients: same sex distribution. Average age was 61 years old, cephalic in $67 \%$ of cases. 53 of patients had lost weight at the time of consultation, $52 \%$ of them evidenced locoregionallymph node involvement and 50\% liver metastases and/or ascites. In 52\% of cases some kind of biliary bypass was performed, surgical in 53\% (63\% pancreaticoduodenectomy), while the rest underwent percutaneous or endoscopic surgery. $31 \%$ of patients did not receive surgical or endoscopic treatment given the advan- 
ced stage of the condition. Average survival of surgical or endoscopic treatment was 3.7 months.

Conclusions: at the time of diagnosis, $50 \%$ of the cases of pancreatic cancer presented metastases and severe nutritional compromise, evidencing locoregional indicators of unresectability or incurability. One third of the cases did not receive oncological treatment given the advanced stage of the disease. Global survival was low. All of these findings suggest it is essential to devise health policies for an early diagnosis and to increase resectability rates.

\section{Resumo}

Introdução: o câncer de pâncreas se caracteriza por sua agressividade biológica, mal prognóstico oncológico e diagnóstico tardio. A maioria tem baja taxa de ressecabilidade ( $10 \%$ a $20 \%)$, devido a presença de fatores específicos que o impedem no momento do diagnóstico.

Objetivo: descrição epidemiológica das características do câncer de pâncreas irresecable, os tratamentos paliativos realizados, a sobrevida global e em relação aos procedimentos realizados.

Material e método: estudo descritivo, multivariável, retrospectivo dos cânceres de pâncreas irresecables, atendidos no período janeiro de 2010 a dezembro de 2015, na Clínica Quirúrgica “3” do Hospital Maciel em Montevidéu, Uruguai.

Resultados: os 30 pacientes com câncer de pâncreas irressecáveis apresentavam uma distribuição igual por sexo, idade media: 61 anos, cefálicos em $67 \%$ dos casos. $53 \%$ dos pacientes haviam emagrecido no momento da primeira consulta; $52 \%$ apresentavam compromisso ganglionar locorregional e 50\% metástases hepáticas e/ou ascite. $52 \%$ dos pacientes foram submetidos a algum tipo de derivação biliar; cirúrgica em 53\% (63\% coledocoduodenostomias) e nos demais percutânea ou endoscópica. $31 \%$ dos pacientes não recebeu tratamento cirúrgico nem endoscópico devido ao estado avançado da doença. A sobrevida media do tratamento paliativo cirúrgico ou endoscópico foi de 3,7 meses.

Conclusões: no momento do diagnóstico do câncer de pâncreas a metade dos pacientes apresentavam metástases e repercussão nutricional severa, com indicadores locorregionais de irressecabilidade ou incurabilidade. Um terço dos casos não recebeu tratamento oncológico devido ao estado avançado da doença. A sobrevida global foi curta. Isto nos permite sugerir que são imprescindíveis medidas sanitárias para seu diagnóstico precoce e aumento da taxa de ressecabilidade.

\section{Bibliografía}

1. Uruguay. Comisión Honoraria de Lucha Contra el Cáncer. V Atlas de mortalidad por cáncer en Uruguay: período
2009-2013. Por sitio primitivo del cáncer. Disponible en: http://www.comisioncancer.org.uy/uc_428_1.html. [Consulta: 14 julio 2017].

2. Winter JM, Cameron JL, Campbell KA, Arnold MA, Chang DC, Coleman J, et al. 1423 pancreaticoduodenectomies for pancreatic cancer: A single-institution experience. J GastrointestSurg 2006; 10(9):1199-210; discussion 1210-1.

3. SharmaC, Eltawil KM,Renfrew PD, Walsh MJ, Molinari M. Advances in diagnosis, treatment and palliation of pancreatic carcinoma: 1990-2010. World J Gastroenterol 2011; 17(7):867-97.

4. Zuckerman DS, Ryan DP. Adjuvant therapy for pancreatic cancer: a review. Cancer 2008; 112(2):243-9.

5. Shi HJ, Jin C, Fu DL. Preoperative evaluation of pancreatic ductal adenocarcinoma with synchronous liver metastasis: diagnosis and assessment of unresectability. World J Gastroenterol 2016; 22(45):10024-37.

6. Lopez NE, Prendergast C, Lowy AM. Borderline resectable pancreatic cancer: definitions and management. World $\mathrm{J}$ Gastroenterol 2014; 20(31):10740-51.

7. Karabicak I, Satoi S, Yanagimoto H, Yamamoto T, Hirooka S, Yamaki S, et al. Risk factors for latent distant organ metastasis detected by staging laparoscopy in patients with radiologically defined locally advanced pancreatic ductal adenocarcinoma. J Hepatobiliary Pancreat Sci 2016; 23(12):750-5.

8. Choi BI, Chung MJ, Han JK, Han MC, Yoon YB. Detection of pancreaticadenocarcinoma: relative value of arterial and late phases of spiral CT. AbdomImaging 1997;22(2):199-203.

9. CTisus. Pancreas: tumors, CT Angiography of Pancreatic Cancer. Disponible en: http://www.ctisus.com/learning/features/syllabus\%20main/pancreas-tumors-ct-angiography-of-pancreatic. [Consulta: 14 julio 2017].

10. National Cancer Institute. Surveillance, epidemiology, and End Results Programs. Cancer stat facts: pancreas cancer. Bethesda, MD: SEER, 2017. Disponible en: https://seer.cancer.gov/statfacts/html/pancreas.html. [Consulta: 14 julio 2017].

11. Barugola G, Partelli S, Marcucci S, Sartori N, Capelli P, Bassi C, et al. Resectable pancreatic cancer: who really benefits from resection? Ann Surg Oncol 2009; 16(12):3316-22.

12. Hartwig W, Hackert T, Hinz U, Gluth A, Bergmann F, Strobel O, et al. Pancreatic cancer surgery in the new millennium: better prediction of outcome. Ann Surg 2011; 254(2):311-9.

13. Tachezy M, Bockhorn M, Gebauer F, Vashist YK, Kaifi JT, Izbicki JR. Bypass surgery versus intentionally incomplete resection in palliation of pancreatic cancer: is resection the lesser evil? J Gastrointest Surg 2011; 15(5):829-35.

14. Müller MW, Friess H, Köninger J, Martin D, Wente MN, Hinz U, et al. Factors influencing survival after bypass procedures in patients with advanced pancreatic adenocarcinomas. Am J Surg 2008;195(2):221-8. 
15. Allen VB, Gurusamy KS, Takwoingi Y, Kalia A, Davidson BR. Diagnostic accuracy of laparoscopy following computed tomography (CT) scanning for assessing the resectability with curative intent in pancreatic and periampullary cancer. Cochrane Database Syst Rev 2016; 7:CD009323.

16. Shepherd HA, Royle G, Ross AP, Diba A, Arthur M, Colin-Jones D. Endoscopic biliary endoprosthesis in the palliation of malignant obstruction of the distal common bile duct: a randomized trial. Br J Surg 1988; 75(12):1166-8.

17. Andersen JR, Sørensen SM, Kruse A, Rokkjaer M, Matzen P. Randomised trial of endoscopic endoprosthesis versus operative bypass in malignant obstructive jaundice. Gut 1989; 30(8):1132-5.

18. Smith AC, Dowsett JF, Russell RC, Hatfield AR, Cotton PB. Randomised trial of endoscopic stenting versus surgical bypass in malignant low bileduct obstruction. Lancet 1994; 344(8938):1655-60.

19. Morizane C, Okusaka T, Morita S, Tanaka K, Ueno H, Kondo S, et al. Construction and validation of a prognostic index for patients with metastatic pancreatic adenocarcinoma. Pancreas 2011; 40(3):415-21

20. Forssell H, Wester M, Akesson K, Johansson S. A proposed model for prediction of survival based on a follow-up study in unresectable pancreatic cancer. BMJ Open 2013; 3(12): 0004064

21. Weber A, Kehl V, Mittermeyer T, Herberich E, Röthling N, Schmid RM, et al. Prognostic factors for survival in patients with unresectable pancreatic cancer. Pancreas 2010; 39(8):1247-53.

22. Alonso Lárraga JO. Tratamiento de la obstrucción biliar en cáncer de páncreas. Rev Gastroenterol Méx 2012; 77(Supl 1):65-6.

23. Soderlund C, Linder S. Covered metal versus plastic stents for malignant common bile duct stenosis: a prospective, randomized, controlled trial. Gastrointest Endosc 2006; 63(7):986-95

24. Moss AC, Morris E, Leyden J, MacMathuna P. Do the benefits of metal stents justify the costs? A systematic review and meta-analysis of trials comparing endoscopic stents for malignant biliary obstruction. Eur J Gastroenterol Hepatol 2007; 19(12):1119-24.

25. Sarr MG, Cameron JL. Surgical management of unresectable carcinoma of the pancreas. Surgery 1982; 91(2):123-33.

26. Sarfeh IJ, Rypins EB, Jakowatz JG, Juler GL. A prospective, randomized clinical investigation of cholecystoenteros- tomy and choledochoenterostomy. Am J Surg 1988; 155(3):411-4.

27. Jacobs PP, van der Sluis RF, Wobbes T. Role of gastroenterostomy in the palliative surgical treatment of pancreatic cancer. J Surg Oncol 1989; 42(3):145-9.

28. Jung GS, Song HY, Kang SG, Huh JD, Park SJ, Koo JY, et al. Malignant gastroduodenal obstructions: treatment by means of a covered expandable metallic stent-initial experience. Radiology 2000; 216(3):758-63.

29. Adler DG, Baron TH. Endoscopic palliation of malignant gastric outlet obstruction using self-expanding metal stents: experience in 36 patients. Am J Gastroenterol 2002; 97(1):72-8.

30. Kneuertz PJ, Cunningham SC, Cameron JL, Torrez S, Tapazoglou N, Herman JM, et al. Palliative surgical management of patients with unresectable pancreatic adenocarcinoma: trends and lessons learned from a large, single institution experience. J Gastrointest Surg 2011; 15(11):1917-27.

31. Siegel R, Naishadham D, Jemal A. Cancer statistics, 2013. CA Cancer J Clin 2013; 63(1):11-30.

32. Truty MJ, Thomas RM, Katz MH, Vauthey JN, Crane C, Varadhachary GR, et al. Multimodality therapy offers a chance for cure in patients with pancreatic adenocarcinoma deemed unresectable at first operative exploration. J Am Coll Surg 2012; 215(1):41-51; discussion 51-2.

33. Von Hoff DD, Ervin T, Arena FP, Chiorean EG, Infante $\mathbf{J}$, Moore M, et al. Increased survival in pancreatic cancer with nab-paclitaxel plus gemcitabine. N Engl J Med 2013; 369(18):1691-703.

34. Conroy T, Desseigne F, Ychou M, Bouché O, Guimbaud R, Bécouarn Y, et al; Groupe Tumeurs Digestives of Unicancer; PRODIGE Intergroup. FOLFIRINOX versus gemcitabine for metastatic pancreatic cancer. N Engl J Med 2011; 364(19):1817-25

35. Okusaka T, Ikeda M, Fukutomi A, Ioka T, Furuse J, Ohkawa S, et al. Phase II study of FOLFIRINOX for chemotherapy-naïve Japanese patients with metastatic pancreatic cancer. Cancer Sci 2014; 105(10):1321-6.

36. Jemal A, Siegel R, Ward E, Murray T, Xu J, Thun MJ. Cancer statistics, 2007. CA Cancer J Clin 2007; 57(1):43-66.

37. Kim HW, Lee JC, Paik KH, Lee YS, Hwang JH, Kim J. Initial metastatic site as a prognostic factor in patients with stage IV pancreatic ductal adenocarcinoma. Medicine (Baltimore) 2015; 94(25):e1012. 